\title{
Relaxation of high-energy quasiparticle distributions: electron-electron scattering in a two-dimensional electron gas
}

\author{
R.N.Gurzhi, ${ }^{1}$ A.I.Kopeliovich, ${ }^{1}$ A.N.Kalinenko, ${ }^{1}$ A.V.Yanovsky,${ }^{1}$ \\ E.N.Bogachek, ${ }^{2}$ Uzi Landman, ${ }^{2}$ H. Buhmann, ${ }^{3}$ and L.W. Molenkamp ${ }^{3}$ \\ ${ }^{1}$ B. Verkin Institute for Low Temperature Physics 8 Engineering, \\ National Academy of Sciences of Ukraine, $4^{7}$ Lenin Ave, Kharkov, 61103, Ukraine \\ ${ }^{2}$ School of Physics, Georgia Institute of Technology, Atlanta, GA 30332-0430, USA \\ ${ }^{3}$ Physikalisches Institut der Universität Würzburg, D-97047 Würzburg, Germany
}

\begin{abstract}
A theory is developed for the evolution of the non-equilibrium distribution of quasiparticles when the scattering rate decreases due to particle collisions. We propose a "modified one-collision approximation" which is most effective for high-energy quasiparticle distributions. This method is used to explain novel measurements of the non-monotonic energy dependence of the signal of scattered electrons in a $2 \mathrm{D}$ system. The observed effect is related to a crossover from the ballistic to the hydrodynamic regime of electron flow.
\end{abstract}

PACS numbers: 72.10.Bg, 73.40.-c, 72.20.My

\section{INTRODUCTION}

The propagation of an electron beam in a twodimensional degenerate electron gas (2DEG) can be used to study the role of electron-electron (e-e) collisions in

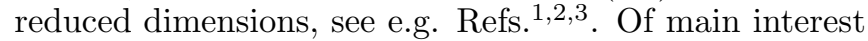
are "high-energy" beams with a typical electron energy that exceeds considerably the thermal energy of the equilibrium electrons within the 2DEG, but remains significantly below the Fermi energy. A special combination of ac- and dc-measurement techniques allows for an energy selective investigation of electrons traversing a defined distance within a 2DEG 4 . Some of these experiments were analyzed in terms of the $\tau$-approximation ${ }^{5.6}$, where it is supposed that each collision scatters an electron out of the beam. However, this approach is obviously insufficient for the case of electron-electron (ee) scattering in a 2DEG, where small angle scattering events dominate, and scattered electrons may remain within the region of the electron beam ${ }^{4,7.899,10}$.

For an adequate theoretical description of ee-scattering it is necessary to take into account the incoming terms in the collision operator $\hat{J}$ (see the second term on the right of the equation for $\hat{J}$ ) of the dynamic integro-differential linearized Boltzmann equation:

$$
\begin{gathered}
\frac{d f}{d t}=\hat{J} f, \\
\hat{J} f=-\nu f+\int d \mathbf{p}^{\prime} \nu_{\mathbf{p p}^{\prime}} f\left(\mathbf{p}^{\prime}\right), \nu=\int d \mathbf{p}^{\prime} \nu_{\mathbf{p}^{\prime} \mathbf{p}}, \\
\frac{d f}{d t} \equiv \frac{\partial f}{\partial t}+\mathbf{v} \frac{\partial f}{\partial \mathbf{r}}+\mathbf{F} \frac{\partial f}{\partial \mathbf{p}} .
\end{gathered}
$$

Here, $f$ is the distribution function of non-equilibrium electrons, $\mathbf{r}$ determines the location of a non-equilibrium electron, and $\mathbf{v}$ and $\mathbf{p}$ are the electron velocity and momentum, respectively (quasi-classical approximation). F is the Lorentz force acting on the electron. The function

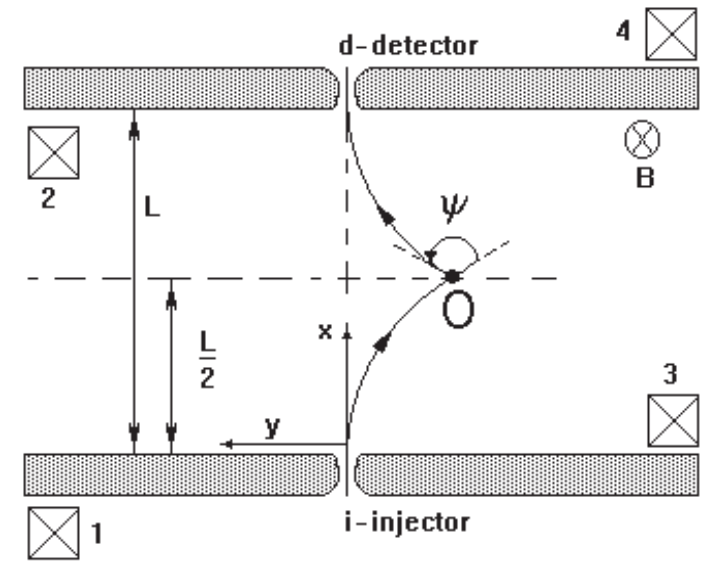

FIG. 1: Scheme of the e-e spectroscopy experiment (see text).

$\nu_{\mathbf{p}^{\prime} \mathbf{p}}$ determines the probability of non-equilibrium electrons $\left(\nu_{\mathbf{p}^{\prime} \mathbf{p}}>0\right)$ or holes $\left(\nu_{\mathbf{p}^{\prime} \mathbf{p}}<0\right)$ to be found in state $\mathbf{p}$ after scattering from state $\mathbf{p}^{\prime}$. A hole in this case describes the absence of an equilibrium electron below the Fermi surface.

In the general case, the solution of the kinetic Boltzmann equation (11) is a very difficult task, both in analytical and numerical calculations. But it is well known from the kinetics of gases that two regimes exist which allow for an essential simplification of the description. First, in the few-collision case the probability is small for an electron to be scattered over a characteristic length scale $L$ ( e.g., the distance between the injector to the detector, see Fig (1 below), which is much smaller than the electronelectron mean-free path, $l$, i.e. $l=v \nu^{-1} \gg L$. Therefore, using perturbation theory it is possible to solve the Boltzmann equation by iteration of the collision operator (quasiballistic approximation). Second, in the case of frequent collisions $(l \ll L)$ a hydrodynamic situation arises. The influence of frequent collisions establishes a quasi-equilibrium distribution which is characterized by 
hydrodynamic variables, such as the average velocity or temperature which depend only smoothly on $\mathbf{r}$. These regimes were studied in our previous publications 4.8.9.10. For obvious reasons a treatment of the intermediate regime $(l \approx L)$ presents considerable difficulties.

In this paper we show that it is possible to study the intermediate regime successfully if the scattering probability decreases substantially with each collision. The occurrence of a decreasing scattering probability can be expressed by an additional small parameter $\Delta$ which is defined as the ratio of the scattering probability of a nonequilibrium electron after a collision to the probability before this event. Such situations can be found in a wide range of problems where non-equilibrium quasiparticles exhibiting some excess energy, above thermal equilibrium, collide with equilibrium quasi-particles and lose a considerable amount of this energy due to this event. Usually, the probability for inelastic scattering decreases drastically with decreasing energy of the quasiparticles, because the statistical weight decreases for both the possibility for finding a collision partner and for finding empty final states. Electron-electron, electronphonon or phonon-phonon collisions at temperatures below the Debye temperature can be taken as an example.

Our approach is based on the following concept: (i) a few collisions are treated exactly, while (ii) subsequent collisions are neglected, or considered perturbationally. We will refer to this approach as the "modified onecollision approximation" (MOC), which accounts for the fact that in most cases a restriction to an one collision approximation is sufficient to explain the general features of the scattering experiments. In the present work, a full account of the MOC approximation is given and the results are applied to analyze experimental data obtained from electron beam experiments. It is shown, that applicability of the MOC approximation to the scattering of high-energy electrons substantially extends the description of spectroscopic data from electron-electron collisions experiments $\stackrel{4}{4}$. The non-monotonic dependence of the signal of scattered electrons on the beam energy can be explained within the MOC approximation. This observation can be interpreted as a crossover from the ballistic to multi-collision (hydrodynamic) flow regime.

\section{MODIFIED ONE-COLLISION APPROXIMATION}

Let us consider non-equilibrium electrons at a characteristic energy $\varepsilon_{0}$, measured from the Fermi level $\varepsilon_{F}$, and larger than the temperature $T$ of the equilibrium electron gas, $\varepsilon_{0} \gg T$. In a first stage of relaxation these highly non-equilibrium electrons are scattered by equilibrium electrons thus generating a new non-equilibrium system but with already much lower excess energy. This stage of relaxation was named the "pretemperature" stage in

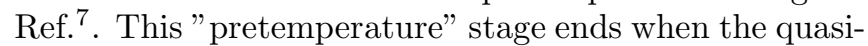
particles energy becomes comparable to the bath temper- ature $T$. The most important fact for the "pretemperature" stage is the following: If the energy $\varepsilon_{0}<\varepsilon_{F}$, then the probability of secondary collision is an order of magnitude lower than for the first one ${ }^{7}$. Indeed, the energy $\varepsilon$ of a non-equilibrium electron after collision with an equilibrium electron will be distributed between three states in phase space:

$$
\begin{aligned}
& \mathbf{p}+\mathbf{p}^{\prime} \rightarrow \mathbf{p}^{\prime \prime}+\mathbf{p}^{\prime \prime \prime} \\
& \varepsilon=\left|\varepsilon^{\prime}\right|+\varepsilon^{\prime \prime}+\varepsilon^{\prime \prime \prime}
\end{aligned}
$$

For $T \ll \varepsilon$, we have $\varepsilon^{\prime}<0$ and $\varepsilon^{\prime \prime}, \varepsilon^{\prime \prime \prime}>0$ due to Pauli principle, i.e. the state $\mathbf{p}^{\prime}$ is below the Fermi level while the final states $\mathbf{p}^{\prime \prime}$ and $\mathbf{p}^{\prime \prime \prime}$ are well above. The energy of the initial electron is redistributed between three final states. On average, the energy of each state after the first collision can be expressed as $\varepsilon_{1} \sim \varepsilon_{0} \cdot \Delta$, with $\Delta^{2} \approx$ $10^{-1}$ (Note, the numerical value of $\Delta$ depends on the specific type of problem considered $)^{7}$. Since the energy dependent mean-free path $l(\varepsilon) \propto 1 / \varepsilon^{2}$, this means that $l(\varepsilon)$ of non-equilibrium quasi-particles will increase by about an order of magnitude, which implies that $l\left(\varepsilon_{1}\right) \approx$ $\Delta^{-2} \cdot l\left(\varepsilon_{0}\right) \gg l\left(\varepsilon_{0}\right)$. This may lead to a situation where the probability for a secondary collision is low while the probability for the first collision is close to unity in a system of size $L: l\left(\varepsilon_{1}\right) \gg L \gtrsim l\left(\varepsilon_{0}\right)$. In this case, it is a natural choice to consider the first collision exactly while the secondary collision is treated by perturbation theory, which leads to an extension of the validity of the one-collision approximation up to $L \sim 10 l\left(\varepsilon_{0}\right)$.

In the following, we use this approach for the case of propagation of nonequilibrium electrons in an electron beam injected into a $2 \mathrm{DEG}$ via a quantum point-contact. For steady state conditions, the operator $d / d t$ on the left side of Eq. (1) must be replaced by $(\mathbf{v} \partial / \partial \mathbf{r})+(\mathbf{F} \partial / \partial \mathbf{p})$. For further consideration it is convenient to use Cartesian coordinates. The $\mathrm{x}$-axis is along the beam direction, while the origin is located at the center of the electron beam injector (a quantum point-contact in an actual experiment). Eq. (1) will be solved by integrating along the trajectory. We define $t$ as the time of motion of the particle along a given trajectory. $\mathbf{R}(\mathbf{r}, \mathbf{p}, t)$ is the (Lagrange) coordinate of the particle at a time $t$. At the time of observation (taken as the origin of the time counting), the particle is located at the point $\mathbf{r}$ with a momentum $\mathbf{p}$. The momentum of the particle at a time $t$ is $\mathbf{P}(\mathbf{r}, \mathbf{p}, t)=m \mathbf{V}=m(\partial \mathbf{R} / \partial t)$. The particle trajectory $\mathbf{R}(\mathbf{r}, \mathbf{p}, t)$ is uniquely defined for given applied fields. The solution of the equation $X\left(\mathbf{r}, \mathbf{p}, t_{0}\right)=0$ defines the start time $t_{0}$ of the electron injection into the system at $x=0$. The solution of Eq. (11) is given as

$$
f=\sum_{n=0}^{\infty} f^{(n)},
$$




$$
\begin{gathered}
f^{(n+1)}(\mathbf{r}, \mathbf{p})=\int_{t_{0}}^{0} d t \int d \mathbf{p}^{\prime}\left[\nu_{\mathbf{P} \mathbf{p}^{\prime}}-\delta\left(\mathbf{P}-\mathbf{p}^{\prime}\right) \nu\right] f^{(n)}\left(\mathbf{R}, \mathbf{p}^{\prime}\right) \\
\equiv \int_{t_{0}}^{0} d t(\hat{\nu}-\nu) f^{(n)}
\end{gathered}
$$

In this expression each $n$-th term of the series is obtained by $n$ subsequent actions of the collision operator on the initial or unpertubated function $f^{(0)}$ which describes the ballistic motion of the particle:

$$
\begin{aligned}
f^{(0)}(\mathbf{r}, \mathbf{p}) & =f_{0}\left(Y_{0}, \mathbf{P}_{0}\right), \\
Y_{0} & =Y\left(\mathbf{r}, \mathbf{p}, t_{0}\right), \\
\mathbf{P}_{0} & =\mathbf{P}\left(\mathbf{r}, \mathbf{p}, \mathbf{t}_{\mathbf{0}}\right)
\end{aligned}
$$

Here, $f_{0}(y, \mathbf{p})$ is an injected distribution where all the injected electrons have a characteristic energy $\varepsilon_{0}$. The quasi-particles, which appear as the result of a collision, are described by the action of the incoming part $\hat{\nu}$ of the collision operator $\hat{J}$ on $f_{0}(y, \mathbf{p})$. In other words, $\hat{\nu} f_{0}$ correspond to a particle that has been scattered once, while $\hat{\nu} \hat{\nu} f_{0}$ describes a subsequent second scattering event, and so forth. Correspondingly, the typical energies of these states differ: $f_{0}$ is characterized by the energy $\varepsilon_{0}$, for $\hat{\nu} f_{0}$ the characteristic energy will be $\varepsilon_{1} \sim \varepsilon_{0} \cdot \Delta$ and for $\hat{\nu} \hat{\nu} f_{0}$ it will be given by $\varepsilon_{2} \sim \varepsilon_{0} \cdot \Delta^{2}$ etc. Consequently we find that $v^{-1} \nu \hat{\nu} f_{0} \sim l^{-1}\left(\varepsilon_{1}\right) \hat{\nu} f_{0}$ and that $\hat{\nu} v^{-1} \nu f_{0} \sim l^{-1}\left(\varepsilon_{0}\right) \hat{\nu} f_{0}$. Therefore, as a main approximation in the parameters $l\left(\varepsilon_{0}\right) / l\left(\varepsilon_{1}\right), L / l\left(\varepsilon_{1}\right) \ll 1$, it is possible to neglect all terms in the series of Eq. (III) that include $\hat{\nu}$ or $\nu$ to an order higher than one, where $\nu$ is placed to the left of $\hat{\nu}$. After making this approximation, the summation of the remaining terms can be carried out easily. Thus, the main idea consists in a partial summation of the infinite series to a first or subsequent approximation, as required in order to reach sufficient accuracy for an adequate description of the experiment.

For a stationary beam in a magnetic field, a Green's function of the "MOC-1" (i.e. the solution of Eq. (11) for $f_{0}(y, \mathbf{p})=\delta(y) \delta\left(\mathbf{p}-\mathbf{p}_{0}\right)$, that takes into account the first collision exactly) has the following form:

$$
g(\mathbf{r}, \mathbf{p})=g_{b}+\sum \nu_{\mathbf{P P}} \frac{V_{0 x}}{\left|\mathbf{V}_{\mathbf{1}} \times \mathbf{V}^{\prime}\right|} \exp \left\{\nu\left(\varepsilon_{0}\right)\left(t_{0}-t^{\prime}\right)\right\} .
$$

Here $g_{b}$ is the distribution of the ballistic part of the electrons, i.e., the solution of Eq. (11) in the absence of the integral operator $\hat{\nu} . t^{\prime}$ is the time of collision and $t_{0}$ is the starting time. $\mathbf{P}^{\prime}=m \mathbf{V}^{\prime}$ and $\mathbf{P}_{\mathbf{1}}=m \mathbf{V}_{\mathbf{1}}$ are the momenta before and after collision, respectively, while $\mathbf{V}_{0}=\mathbf{P}_{\mathbf{0}} / m$. The values of $t, t_{0}, \mathbf{R}\left(\mathbf{r}, \mathbf{p}, t^{\prime}\right)$, $\mathbf{P}^{\prime}=\mathbf{P}\left(\mathbf{0}, \mathbf{p}_{\mathbf{0}}, \mathbf{t}_{\mathbf{0}}-\mathbf{t}^{\prime}\right), \mathbf{P}_{\mathbf{1}}=\mathbf{P}\left(\mathbf{r}, \mathbf{p}, t^{\prime}\right)$, and $\mathbf{p}$ are determined through the equations of motion by the initial momentum $\mathbf{p}_{0}$ and location $\mathbf{r}$. The equations of motion are reduced to $\mathbf{R}\left(\mathbf{r}, \mathbf{p}, t^{\prime}\right)=\mathbf{R}\left(0, \mathbf{p}_{0}, t_{0}-t^{\prime}\right)$. The presence of walls in the experimental setup, see Fig. 1 excludes solutions with a full cyclotron orbit.
Note that the MOC approximation is applicable in principle under conditions, where a pre-temperature stage occurs for the non-equilibrium quasi-particles. In other words, it holds when the following hierarchy of mean-free paths is given:

$$
l\left(\varepsilon_{0}\right) \ll l\left(\varepsilon_{1}\right) \ll l\left(\varepsilon_{2}\right) \ll l\left(\varepsilon_{3}\right) \ll \ldots \lesssim l(T),
$$

where $\varepsilon_{i}$ is the energy after the $i$-th collision and $l\left(\varepsilon_{0}\right) \lesssim$ $L \ll l(T)$; for $L \ll l\left(\varepsilon_{0}\right)$ the inequality corresponds to the quasi-ballistic regime, where perturbation theory is applicable. If $L$ corresponds to the $n$-th segment of this hierarchy, i.e. $l\left(\varepsilon_{n}\right)<L<l\left(\varepsilon_{n+1}\right)$, it is necessary to construct a "MOC- $n$ " approximation by treating the first $n$ collisions exactly; which leads to relative corrections of the order of $\Delta^{2}$. The approach breaks down when the quasi-particle energy is comparable to $T$. As an example, the exact result for the first two collisions ("MOC-2"), for the spatially homogeneous case and $f_{0} \propto \delta\left(\varepsilon-\varepsilon_{0}\right)$, reads:

$$
\begin{array}{r}
f=\left\{\exp \left(-\nu_{0} t\right)+\left(\nu_{0}-\nu\right)^{-1}\left(\exp \left(-\frac{\nu t}{1-\nu / \nu_{0}}\right)\right.\right. \\
\left.-\exp \left(-\nu_{0} t\right)\right) \hat{\nu}+\nu_{0}^{-1} \hat{\nu}\left[\nu^{-1}(1-\exp (-\nu t))\right. \\
\left.\left.+\left(\nu_{0}-\nu\right)^{-1}\left(\exp \left(-\nu_{0} t\right)-\exp \left(\frac{\nu t}{1-\nu / \nu_{0}}\right)\right)\right] \hat{\nu}\right\} f_{0} \\
\nu_{0}=\nu\left(\varepsilon_{0}\right) .
\end{array}
$$

This formula is valid for times $t<l\left(\varepsilon_{2}\right) / v_{F}$. Thus, just a few steps lead to a high accuracy. It turns out, that for the experiments discussed below, just one step is enough to produce satisfactory results.

\section{EXPERIMENT}

In order to investigate the electron-electron scattering properties in a 2DEG, experiments are needed which allow to distinguish between scattered and non-scattered particles, and reveal the details of scattered signal. Therefore, we used the experimental setup of the electron spectroscopy method proposed in Ref ${ }^{4}$ (see Fig. 1). The sample is based on a conventional Si-modulation doped $\mathrm{Ga}-\mathrm{As}(\mathrm{Al}, \mathrm{Ga})$ As heterojunction with a carrier density of $n \approx 2.8 \cdot 10^{11} \mathrm{~cm}^{-2}$ and an impurity mean-free path of $l_{i m} \approx 20 \mu \mathrm{m}$. The scheme of the experiment is shown in Fig. 1] Schottky gates define quantum point-contacts in the 2DEG and are adjusted such that the injector and detector quantum point-contact (QPC) are carrying only one conducting mode. The distance between the injector and detector QPC is $L=4 \mu \mathrm{m}$. Ohmic contacts are indicated by crossed squares in Fig. [1 The sample was cooled down to $T=0.2 \mathrm{~K}$. An ac-modulated dc-bias voltage applied between ohmic contacts 1 and 2 was used to inject the electrons into the 2DEG area between injector and detector. The amplitude of the ac modulation $\delta V_{i}$ was much smaller than the dc component, $\delta V_{i} \ll V_{i}$. The 


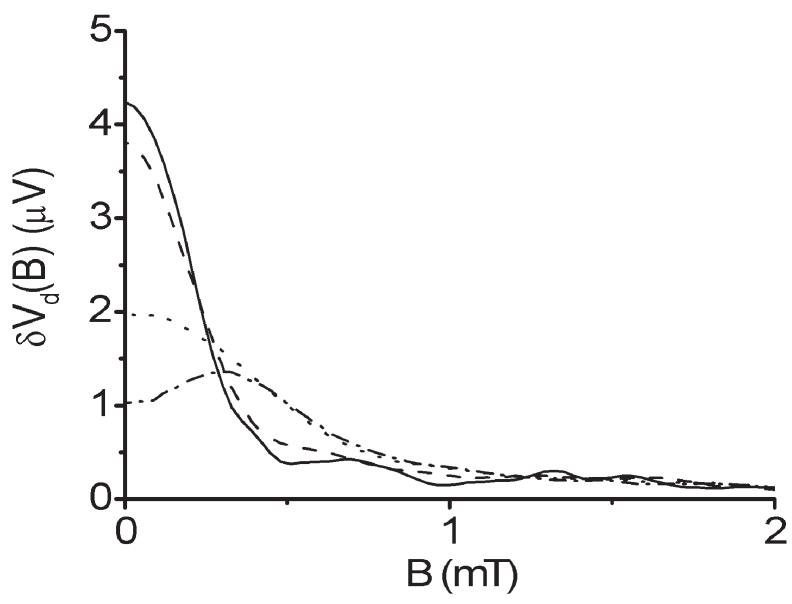

FIG. 2: The experimentally measured signal on the detector as a function of magnetic field for different energies of injection: (solid) $-0.8 \mathrm{mV}$, (dash) - $1.6 \mathrm{mV}$, (dot) $-2.6 \mathrm{mV}$, (dash-dot) $-3.4 \mathrm{mV}$.

ac-component of the detector signal, i.e. the voltage that occurs between contacts 3 and $4\left(V_{d}=V_{34}\right)$ is measured with a lock-in technique. With this technique only the signal of electrons with energy $V_{i}$ is measured, while the whole e-beam consists of electrons with energies in the range of $0 \ldots V_{i}$. (Note that, throughout this paper the electron charge $e$ is set equal to one $e=1$.)

The magnetic field dependence of the detector signal $\delta V_{d}\left(B, V_{i}\right)$ has been studied for a wide range of energies $V_{i}=0 \ldots 9 \mathrm{mV}$. An example is given in Fig. 2 for different injection energies $V_{i}$. This figure display the evolution of an mono-energetic electron beam with increasing injection energy $V_{i}$, which reflects the energy-dependent electron-electron scattering. From the half-hight width of low energy curve $(1)\left(V_{i}=01 . \mathrm{mV}\right)$ it is possible to extract the opening angle of the collimated electron beam: $\Phi \approx 12^{\circ}$, which is identical for injector and detector when the QPCs are equally adjusted to one conducting mode $\left(G_{\mathrm{QPC}}=2 / h\right)$.

The contribution of scattered particles to the detector signal $\delta V_{d}^{s}$ can be easily extracted following the approach of Ref. ${ }^{4}$ :

$\delta V_{d}^{s}\left(B, V_{i}\right)=\delta V_{d}\left(B, V_{i}\right)-\exp \left(-\frac{2 r_{c}}{l\left(V_{i}\right)} \arcsin \frac{L}{2 r_{c}}\right) \delta V_{d}^{0}(B)$,

here, $l\left(V_{i}\right)=4 \pi \hbar v_{F}\left(\varepsilon_{F} / V_{i}\right)^{2} / \varepsilon_{F}\left(\ln \varepsilon_{F} / V_{i}+\ln 2 q_{T F} / k_{F}+\right.$ $1 / 2)$ is the energy dependent e-e scattering length according to Ref. 6 , where $q_{T F}$ is the Thomas-Fermi screening wave vector; $r_{c}$ is the cyclotron radius; $v_{F}$ is the Fermivelocity. The second term on the right-hand side of this expression corresponds to $g_{b}$ defined in Eq. (4), $\delta V_{d}^{0}(B)$, represents the signal that would be observed in the absence of scattering. A good approximation for this value can be obtained from the signal measured for the lowest excitation voltage $\delta V_{d}\left(B, V_{i}\right)$ at $V_{i}=0.1 \mathrm{mV}$. In this

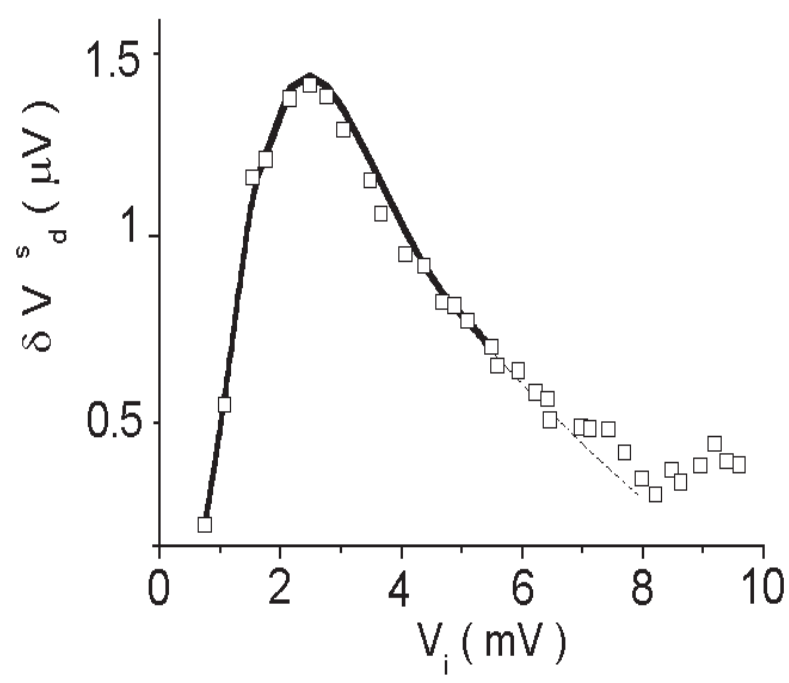

FIG. 3: The maximum of scattered particles signal as a function of injection energy. Empty square markers correspond to the experimental values. The thick line represents the theory. The dashed line represents theoretical calculations outside the range of applicability of the approximation used here.

cases, $L / l \sim 10^{2}$ and electron-electron collisions can be ignored. Note that for injection energies $V_{i}>3.5 \mathrm{mV}$ $\approx \varepsilon_{F} / 3$ the above equation for $l\left(V_{i}\right)$ yields a large inaccuracy. However, this can be ignored because $l\left(V_{i}\right)$ becomes so small that the subtraction of the ballistic contribution to the signal leads only to corrections of less than one percent ${ }^{4}$.

\section{DISCUSSION}

In Fig. 3 the maximum value of the magnetic field dependent signal due to the scattered electrons, $\delta V_{\max }^{s}\left(V_{i}\right)=\max _{B} \delta V_{d}^{s}\left(B, V_{i}\right)$ is presented as a function of the injection energy $\left(V_{i}\right)$. This dependence shows a distinct non-monotonic behavior, i.e. a rapid increase at small $V_{i}$, a distinct maximum at $V_{i} \approx 2 \mathrm{mV}$ and an abrupt decrease for larger injection energies up to $V_{i} \sim 8$ $\mathrm{mV}$, followed by a weak increase.

The presence of a maximum and the subsequent decrease in the energy dependence of $\delta V_{\max }^{s}\left(V_{i}\right)$ can not be explained in the context of a simple perturbation theory applied to the collision integral, because in the regime where the maximum is observed the electron-electron mean-free path is already smaller than the distance between the injector and detector QPC. Perturbation theory can only be applied to describe the increase of the signal for small energies $V_{i}$. The presence of a strong decrease appears at first sight to be unexpected, because in the multi-collision regime that is entered upon increasing $V_{i}$, a decrease of the ac-signal from scattered particles is not anticipated. In this multi-collision regime the ballistic beam is already absent but the motion of the electrons 
can be represented by a hydrodynamic drift.

However, the behavior shown in Fig. 3 can be explained in the context of the modified one-collisional approximation described above by taking into account the specific properties of e-e scattering in two-dimensions ${ }^{7}$. A calculation shows, that by taking into account the first collision exactly, Eq. (4) leads to the following expression for the detector signal due to scattered particles

$\delta V_{d}^{s} \propto \nu K(\psi) G(\psi) \exp \left(-\frac{L}{4 l} \frac{\psi}{\sin \psi / 2}\right), \psi=2 \arcsin \frac{L}{2 r_{c}}$,

where $\psi$ is the scattering angle and $G(\psi)=$ $\int_{-\Phi / 2}^{\Phi / 2} d \varphi g(\psi-\varphi)$ is the angular distribution function of e-e scattering, $g(\psi)=m \nu^{-1} \int d \varepsilon \nu_{\varepsilon \varepsilon_{0}}(\psi)$, averaged over an interval $\Phi$; the weighting coefficient $K$ is $K \approx 1 / \Phi$ for $\psi \lesssim \Phi$ and $K \approx 1 / \sin \psi$ for $\Phi<\psi<\pi / 2$. (It is sufficient to consider only the case where the cyclotron radius $r_{c}$ is smaller than $L$.) It should be noted that Eq. (8) is valid for all ratios of $\Phi$ and the angular width of $g(\psi)$. The expression $L \psi / 4 \sin (\psi / 2)$ in the exponent of Eq. (8) represents the length of the electron trajectory from the injector to the point $O$ where the scattering takes place (see Fig. 1). From Eq. (8) it follows, that the signal due to scattered particles is directly proportional, on the one hand, to the e-e scattering probability, or the frequency of the collisions $\nu$ and, on the other hand, it is proportional to the probability of an electron to reach the scattering point $O$ ballistically. As has been shown earlier in ${ }^{4}$, the pre-exponential factor increases with increasing $V_{i}$ at low injection energies (mainly due to the increase of the collision frequency $\left.\nu \propto V_{i}^{2}\right)$.

The existence of a maximum in Fig. [3]can be attributed to a competition between an increase of the scattering probability (the pre-exponential factor) and a decrease of ballistic transport into the neighborhood of the scattering point $O$ (the exponent) with $V_{i}$. This explanation is supported by the excellent quantitative agreement between the experimental data and a theoretical fit based on Eq. (8]), where the angle $\psi$ is replaced by the experimental value $\psi_{\max }$, see Fig. 3

The good fit also justifies the use of the one-collision MOC approximations indicating that higher orders collisions can be neglected. For small energies, the preexponential factor determines the behaviour of the signal due to scattered particles, which also can be described by standard perturbation theory applied to the collisions integral. Around the maximum the probability for secondary collisions is still small and the signal is still defined by the scattering that takes place in the neighborhood of the point $O$ - however, the probability for a first collision on the way between the injector and the detector becomes larger. This underlines the decrease of the signal with increasing $V_{i}$. As mentioned above this regime can not be described by ordinary perturbation theory, requing the MOC approximation. At this point we would like to note that the non-monotonic behavior of the ac- current component which has been discussed above can not be explained by nonlinearities due to heating effects which are expected to appear at approximately the same values of $V_{i}$ in dc-measurements ${ }^{9}$. It can be shown, that such an effect gives only a small correction of the order of $T\left(V_{i}\right) / V_{i}$ to the ac-component of the signal, where $T\left(V_{i}\right)$ is the temperature due to the heating effect.

Finally, we may only speculate about the origin of the weak increase of the detector signal which is observed for the highest injection energies $V_{i}$. As the injection energy approaches $\varepsilon_{F}$, the potential drop across the injector QPC becomes so large that the second subband of the QPC may become populated. This enhances the injector conductance, and, consequently, the detected signal. For these energies Eq. (8) is no longer valid (see Ref ${ }^{4}$ ). A second reason could be transition to the two-collision (or multi-collision) regime as the mean-free path is less then $10^{-1} \mathrm{~L}$ at these energies.

\section{CONCLUSION}

In summary, we have shown in this paper that it is possible to successfully investigate the evolution of a highenergy non-equilibrium quasi-particles distribution with the help of the "modified one-collision approximation". This method was used to explain novel measurements of the non-monotonic energy dependence of the signal of scattered electrons in a $2 \mathrm{D}$ system. We have obtained an excellent quantitative agreement between the experimental data and a theoretical calculation.

In conclusion we would like to emphasize that the MOC approximation may be of interest for a wide range of problems independent of the nature of the scattering particles; the only restriction is that the scattering probability should strongly decrease after one or several collisions. Along with the case of electron-electron scattering, this is a typical situation for the majority of inelastic scattering processes, such as electron-phonon, phononphonon etc. Let us discuss as an example, electronphonon scattering in the case when the electron energy is high enough $\varepsilon \gg T$, but is low in comparison with the Debye temperature (the electron energy is measured from the Fermi level). In this case a phonon with an energy of the order of $\varepsilon$ will be emitted as a result of the electronphonon scattering event and the corresponding mean-free path is $l \propto \varepsilon^{-3}$. In contrast to electron-electron scattering the energy is redistributed here between two states according to the momentum and energy conservation laws, $\mathbf{p}_{\mathbf{0}}=\mathbf{p}_{\mathbf{1}}+\mathbf{q}$ and $\varepsilon_{0}=\varepsilon_{1}+\omega$. Here $\mathbf{p}_{\mathbf{0}}$ and $\varepsilon_{1}$ are the quasimomentum and energy before the collision, and after the collision they are $\mathbf{p}_{\mathbf{1}}$, and $\varepsilon_{1}$, respectively; $\mathbf{q}$ is quasimomentum of the emitted phonon and $\omega$ its energy. Since, on average, the energy is redistributed equally between the scattering product $\omega \sim \varepsilon_{1}$, we also have here the small parameter $l\left(\varepsilon_{0}\right) / l\left(\varepsilon_{1}\right) \sim 2^{-3} \simeq 0.1$. The case of three-particles high-energy phonon-phonon scattering processes differs only by the energy dependence of the 
mean-free path.

Furthermore, the inelasticity, on which the approach developed in this paper is based, is likely to be relevant not only to the energy redistribution but also to some other quantities; this includes, for example, the spin in cases where the probability of scattering decreases significantly after a spin-flip scattering event. This case may be realized for electron scattering from spin-polarized impurities (there are no spin subbands in this case) and if the exchange interaction depends significantly on the mutual orientation of the electron spin and the impurity spin. Let us neglect the interaction between electrons of one of the spin directions (spin-up, for example) and the impurities. Then the spin relaxation process is completed just after the first spin-flip scattering of the spin-down electrons. Thus, it is straightforward to describe this process in the framework of the modified one-collision approximation.

The method we proposed in this work can be very effective also for point-contact spectroscopy ${ }^{11,12}$. The reason is the following. The current-voltage characteristic of the electric circuit containing a microconstriction is determined by the back-scattering due to inelastic electron scattering processes just in the region of the microconstriction. If $l(\varepsilon) \lesssim L$ ( $L$ is the length of the constriction) the current-voltage characteristic is determined only by one, or few, first collisions, the probability of which is not small and, thus, it can not be taken into account using a perturbation theory (as it was done in the study described in Ref ${ }^{12}$ for the case when $\left.l(\varepsilon) \gg L\right)$. The same also holds for the nonlinear thermoelectric phenomenon in point-contacts 13 . The influence of the first collisions on the point-contact spectrum may be calculated exactly using equations of the general form of Eq.(6) because in point-contact spectroscopy the excess energy of the electron is high, $\varepsilon \gg T$.

\section{Acknowledgement}

The research described in this publication was made possible in part by Award No.UP2-2430-KH-02 of the U.S. Civilian Research \& Development Foundation for the Independent States of the Former Soviet Union (CRDF)(R.N.G, A.N.K, A.I.K., A.V.Y., E.N.B., and U.L.), by the US Department of Energy Grant FG0586ER-45234 (E.N.B. and U.L.), and by the Deutsche Forschungsgemeinschaft SFB 410 (H.B. and L.M.).
1 L.W. Molenkamp, M.J. Brugmans, H. van Houten and C.T. Foxon, Semicond. Sci. Technol. 7, B228, (1992).

2 Th.Schäpers, M.Krüger, J.Appenzeller, A.Förster, B.Lengeler, and H.Lüth, Appl. Phys. Lett. 66, 3603 (1995).

3 D.R.S. Cumming and J.H. Davis, Appl. Phys. Lett. 69, 3363 (1996).

4 A.V. Yanovsky, H. Predel, H. Buhmann, R.N. Gurzhi, A.N. Kalinenko, A.I. Kopeliovich, L.W. Molenkamp, Europhys. Lett., 56, 709, (2001).

5 A.V. Chaplik, Zh. Eksp. Teor. Fiz., 60,1845 (1971).

${ }^{6}$ G.F. Giuliani, and J.J. Quinn, Phys.Rev. B, 26, 4421 (1982).

7 R.N. Gurzhi, A.N. Kalinenko, A.I. Kopeliovich, Fiz. Nizk. Temp., 23, 58, (1997) [Low Temp. Phys. 23, 44, (1997)]; Surface Science. 361/362, 497, (1995).

8 R.N. Gurzhi, A.N. Kalinenko, A.I. Kopeliovich, Phys. Rev.
Lett. , 74, 3872, (1995); Phys. Rev. B, 52, 4744 (1995).

${ }^{9}$ H. Predel, H. Buhmann, R.N. Gurzhi, A.N. Kalinenko, A.I. Kopeliovich, L.W. Molenkamp, and A.V. Yanovsky, Physical Review B, 62, 2057, (2000).

10 H. Buhmann, R.N. Gurzhi, A.N. Kalinenko, A.I. Kopeliovich, L.W. Molenkamp, H. Predel, A.V. Yanovsky, Physica E, 6, 310, (2000).

11 I.K. Yanson, Zh.Eksp.Theor.Fiz. 66, 1035 (1974)[Sov.Phys.JETP 39, 506 (1074)].

12 I.O. Kulik, A.N. Omelyanchuk, and R.I.Shekhter, Fiz. Nizk. Temp., 3, 1543, (1977) [Sov. J. Low Temp. Phys. 3 789, (1977)].

13 E.N. Bogachek, I.O. Kulik, and A.G.Shkorbatov, Fiz. Nizk. Temp., 11, 1189, (1985) [Sov. J. Low Temp. Phys. 11 656, (1985)]. 